\title{
Medication Errors of Nurses and Factors in Refusal to Report Medication Errors Among Nurses in a Teaching Medical Center of Iran in 2012
}

\author{
Davoud Mostafaei ${ }^{1}$; Ahmad Barati Marnani ${ }^{2, *}$; Haleh Mosavi Esfahani ${ }^{3}$; Fatemeh Estebsari \\ ${ }^{4}$; Shiva Shahzaidi ${ }^{5}$; Ensiyeh Jamshidi ${ }^{6}$; Seyed Samad Aghamiri ${ }^{7}$ \\ ${ }^{1}$ Department of Health Economic and Management, School of Public Health, Tehran University of Medical Sciences, Tehran, IR Iran \\ ${ }^{2}$ Department of Health Service, School of Medical Information Management, Health Management and Economics Research Center, Iran University of Medical Sciences, Tehran, IR \\ Iran \\ ${ }^{3}$ Department of Health Services, School of Medical Information Management, Iran University of Medical Sciences, Tehran, IR Iran \\ 4 Department of Health, School of Nursing and Midwifery, Shahid Beheshti University of Medical Sciences, Tehran, IR Iran \\ 5 Shohada Tajrish Hospital, Shahid Beheshti University of Medical Sciences, Tehran, IR Iran \\ ${ }_{7}^{6}$ Research Center of Community Based Participatory, Iranian Institute for Reduction of High-Risk Behaviors, Tehran University of Medical Sciences, Tehran, IR Iran \\ 7 Ran Helal Institute of Applied Sciences and Technology, Tehran, IR Iran \\ ${ }^{*}$ Corresponding Author: Ahmad Barati Marnani, Deptartment of Health Services Management, School of Medical Information Management, Health Management and Economics \\ Research Center, Iran University of Medical Sciences, Tehran, IR Iran. Tel: +98-2188794301, Fax:+98-2188793805, E-mail: a.barati@tuma.ac.ir.
}

Received: December 4, 2013; Revised: June 21, 2014; Accepted: August 30, 2014

\begin{abstract}
Background: About one third of unwanted reported medication consequences are due to medication errors, resulting in one-fifth of hospital injuries.

Objectives: The aim of this study was determined formal and informal medication errors of nurses and the level of importance of factors in refusal to report medication errors among nurses.

Patients and Methods: The cross-sectional study was done on the nursing staff of Shohada Tajrish Hospital, Tehran, Iran in 2012. The data was gathered through a questionnaire, made by the researchers. The questionnaires' face and content validity was confirmed by experts and for measuring its reliability test-retest was used. The data was analyzed by descriptive statistics. We used SPSS for related statistical analyses.

Results: The most important factors in refusal to report medication errors respectively were: lack of medication error recording and reporting system in the hospital (3.3\%), non-significant error reporting to hospital authorities and lack of appropriate feedback (3.1\%), and lack of a clear definition for a medication error (3\%). There were both formal and informal reporting of medication errors in this study. Conclusions: Factors pertaining to management in hospitals as well as the fear of the consequences of reporting are two broad fields among the factors that make nurses not report their medication errors. In this regard, providing enough education to nurses, boosting the job security for nurses, management support and revising related processes and definitions are some factors that can help decreasing medication errors and increasing their report in case of occurrence.
\end{abstract}

Keywords:Medication Error; Reporting System; Nurse

\section{Background}

A medication error is any preventable event that may cause or lead to inappropriate medication use or patient harm; while the medication is in the control of the health care professional, patient, or consumer. Such events may be related to professional practice, health care products, procedures and systems including prescribing, order communication, product labeling, packaging, and nomenclature, compounding, dispensing, distribution, administration, education, monitoring, and use. Medical error is one of the factors causing death and harm to patients and the most common important challenges threatening healthcare system in all countries worldwide (1). Studies indicate that most of the errors occur at the time of prescribing or giving medication to patients (2). Importance of "reporting systems" and "not-blame culture" is known as two approaches can influence medi- cal or medication error. In other words, physicians make prescribing errors; while nurses make an error most often at the time of giving medications to the patients (3). However, studies indicate that although nurses prevent $48 \%$ of medication errors resulted from wrong prescription, errors occurred at the time of giving medications, which consist $28 \%$ of the total errors, are usually made by nurses (4). As far as the medication is given directly to patients, there is no way to prevent the errors (3-5).

In addition, medication errors have adverse consequences such as the rise of mortality rate among patients and increase of length of stay and medical expenses (3). Occurring such errors also makes patients lose their reliance on the system of providing healthcare services and results in their dissatisfaction with the system. It also can result in stress and ethical conflicts of nurses (6). error

Copyright ( ) 2014, Iranian Red Crescent Medical Journal; Published by Kowsar. This is an open-access article distributed under the terms of the Creative Commons Attribution-NonCommercial 4.0 International License (http://creativecommons.org/licenses/by-nc/4.0/) which permits copy and redistribute the material just in noncommercial usages, provided the original work is properly cited. 
in medications have negative effects on the quality of patients caring, the performance of nurses and healthcare centers and results in low quality of care $(2,7)$; however, timely diagnosing the errors as well as taking appropriate strategies for deceasing their occurrences are very important. In this regard, several studies have been conducted to investigate barriers that prevent nurses from reporting. Management factors and fear of the consequences of reporting errors are two important barriers among nurses $(6,7)$.

On the other hand, other individual and organizational factors are reported as barriers to reporting medication errors by nurses, including not knowing or diagnosing whether an error has occurred, the importance of the occurred medication error, the fear of authorities' reaction, the necessary time for gathering documents related to the errors and weak feedback of authorities after reporting the errors $(6,8)$.

Creating a secure environment for the nursing staff after a medical error occurrence can decrease the rate of the errors to the lowest possible level and increase of reporting inevitable errors to senior officials without the fear of consequences. Therefore, we have conducted this study to investigate the most important causes and factors making nurses avoid reporting medication errors when occurring. Previous studies have examined the relationship between demographic variables with medication errors therefor; the aim of this study was determined formal and informal medication errors of nurses and the level of importance of factors in refusal to report medication errors among nurses. Outcome of this study may use for suggesting strategies for appropriate organizational framework for reporting medication errors in hospitals.

\section{Objectives}

The aim of this study was to determine formal and informal medication errors of nurses and the level of importance of factors in refusal to report medication errors among nurses.

\section{Patients and Methods}

\subsection{Study Population and Sampling}

After obtaining the necessary authorizations from the Ethics Committee of Shahid Beheshti University of Medical Sciences (Ethical ID 1944, 5/25/2012) and making necessary coordination with Shohada Tajrish Hospital, Tehran, Iran, this study was conducted on 100 nurses (with at least 2 years work experience in hospital wards) that worked in the hospital. The number of samples was determined based on sample size formula.

\subsection{Data Collection}

We used a questionnaire constructed by the researchers, which consisted of two parts. The first part, includ- ing demographic data, was consisted of seven questions. The second part, consisted of nineteen questions, which measured the number of errors made by nurses and also the number of formal and informal reports of errors of nurses. To determine the Content Validity Index (CVI) and the Content Validity Ratio (CVR), a panel of experts ( $\mathrm{n}=$ 10) reviewed scale items. The CVI and CVR scores of the scale were 0.87 and 0.89 , respectively. For measuring reliability, a test-retest was administered. The questionnaire was distributed among twenty subjects of the population twice with the interval of two weeks. In both times, Cronbach's alpha the reliability coefficient was 0.81 , which indicated the aforementioned questionnaire has an acceptable utility level.

Afterwards, the questionnaires were distributed among nurses in three shifts (morning/afternoon/night). After giving them the necessary explanations about the objectives of the research and the way of answering the items, they were asked to answer the questionnaire in less than three days after receiving them, which was the appropriate time. In addition, to increase response rate and to decrease attrition rate, every questionnaire was delivered along with a colorful folder and a pen.

\subsection{Ethical Consideration}

After receiving necessary authorizations, oral and written consent was obtained from the participants. They were ensured that the data would remain confidential and used for the research purposes only.

\subsection{Data Analysis}

At the next phase, the questionnaires were gathered and after initial control of completeness of questions, answers and coding, the data was entered into SPSS version 18 and then analyzed using appropriate statistical tests. Descriptive statistics was used and the statistical significance level was set on 0.05 .

\section{Results}

At the end of the study, 100 individuals were included, in which 85 (85\%) were female and 15 (15\%) were male. The average age was $31.3+5.2$ years and the average of work experience was $6.4+1.5$ years. The findings also indicate that nursing staff who worked in different shifts was $86 \%$ and only $14 \%$ worked in only one shift.

In terms of employment type, the highest frequent proportion belongs to employees with contractual employment which were $31 \%$, the second place belongs to those with formal and temporary employment, each with $24 \%$ and the last employment type belongs to those with two years' work commitments after graduation, the frequency of which is $21 \%$. Table 1 shows the frequency of formal reporting of medication errors among nurses. The findings indicate that the smallest portion of formal reporting of medication errors among nurses belongs 
to nonpaying attention to proper position of patients regarding the kind of medication, using undiluted drug when diluted drug is needed, the intravenous injection of intramuscular medication and error in the injection method in terms of the speed of injection, respectively. The most frequent cases of formal reported medication errors from the highest to the lowest are: giving post-operative analgesics without physician's prescription (70\%), giving medication without physician's prescription, not paying attention to effects of drug interactions (53\%) and intravenous injection of subcutaneous medications, respectively.

Table 2 demonstrates that the least frequency of informal reporting of medication errors among nurses investigated here belong to not giving the prescribed medication to patients, giving patients sublingual or chewable medications orally, not diluting a medications that have to be diluted, mixing medication in micro set without paying attention to drug interactions, respectively. The most frequent informal reporting of medication errors belong to giving postoperative analgesics without physician's prescription, giving expired medications to patients, lack of attention to proper time of giving medications (before and after food) and error in injection method in terms of speed of injection, respectively.

Given the data in Table 3, the most important factors in refusal to report medication errors among the nurses under investigation are lack of recording system for medication errors and reporting them to hospital authorities, lack of appropriate feedback, and lack of a clear definition for medication errors, respectively. The least important factors in not reporting medication errors among nurses are the fear of facing with legal authorities, the fear of job losing, and fear of consequences and adverse effects of medication errors, respectively. It is worth mentioning that after analyzing the data, there was no significant relationship between demographic variables and the level and the causes of reporting medication errors.

Table 1. Frequency of Formal Reporting Medication Errors of Nurses in the Study

\begin{tabular}{|c|c|c|c|c|c|c|}
\hline \multirow[t]{2}{*}{ No } & \multirow[t]{2}{*}{ Medication Error } & \multicolumn{4}{|c|}{$\begin{array}{l}\text { Frequency of Formal Reporting of Medication } \\
\text { Errors, \% }\end{array}$} & \multirow[t]{2}{*}{ Total } \\
\hline & & Never & Once & Twice & More Than Twice & \\
\hline 1 & Not giving prescribed medication to the patient & 63 & 28 & 6 & 3 & 100 \\
\hline 2 & $\begin{array}{l}\text { Giving medication to the patient without prescription of } \\
\text { physician }\end{array}$ & 53 & 35 & 8 & 4 & 100 \\
\hline 3 & Giving medication before or after due time & 63 & 33 & 0 & 4 & 100 \\
\hline 4 & Not diluting a medication that has to be diluted & 69 & 27 & 4 & 0 & 100 \\
\hline 5 & $\begin{array}{l}\text { Not paying attention to proper time of giving a medication } \\
\text { (before and after food) }\end{array}$ & 66 & 29 & 0 & 2 & 100 \\
\hline 6 & $\begin{array}{l}\text { Not taking necessary measures for medications that need } \\
\text { special attention (taking blood pressure and pulse, etc.) }\end{array}$ & 66 & 29 & 5 & 0 & 100 \\
\hline 7 & $\begin{array}{l}\text { mixing medications (micro set medications) without pay- } \\
\text { ing attention to drug interactions }\end{array}$ & 66 & 29 & 1 & 4 & 100 \\
\hline 8 & Error in injection method in terms of speed of injection. & 68 & 27 & 4 & 1 & 100 \\
\hline 9 & the intravenous injection of an Subcutaneous medication & 66 & 28 & 2 & 4 & 100 \\
\hline 10 & the Subcutaneous injection of an intravenous medication & 66 & 30 & 4 & 0 & 100 \\
\hline 11 & the intramuscular injection of an intravenous medication & 66 & 30 & 1 & 3 & 100 \\
\hline 12 & the intravenous injection of an intramuscular medication & 69 & 25 & 6 & 0 & 100 \\
\hline 13 & giving patients sublingual or chewable medications orally & 67 & 26 & 4 & 3 & 100 \\
\hline 14 & giving expired medications to patients & 67 & 26 & 6 & 1 & 100 \\
\hline 15 & $\begin{array}{l}\text { giving postoperative analgesics without physician's pre- } \\
\text { scription }\end{array}$ & 65 & 29 & 5 & 6 & 100 \\
\hline 16 & Giving the wrong medication to a patient & 67 & 29 & 0 & 4 & 100 \\
\hline 17 & Giving more or less than the prescribed dose & 62 & 32 & 5 & 1 & 100 \\
\hline 18 & $\begin{array}{l}\text { not paying attention to proper position of patients regard- } \\
\text { ing the kind of medication }\end{array}$ & 70 & 25 & 5 & 0 & 100 \\
\hline 19 & not paying attention to effects of drug interactions & 63 & 31 & 2 & 4 & 100 \\
\hline
\end{tabular}




\begin{tabular}{|c|c|c|c|c|c|c|}
\hline \multirow[t]{2}{*}{ No } & \multirow[t]{2}{*}{ Medication Error } & \multicolumn{4}{|c|}{$\begin{array}{c}\text { Frequency of Informal Reporting of } \\
\text { Medication Errors, \% }\end{array}$} & \multirow[t]{2}{*}{ Total } \\
\hline & & Never & Once & Twice & $\begin{array}{l}\text { More Than } \\
\text { Twice }\end{array}$ & \\
\hline 1 & Not giving prescribed medication to the patient & 76 & 23 & 1 & 0 & 100 \\
\hline 2 & Giving medication to the patient without prescription of physician & 39 & 53 & 8 & 0 & 100 \\
\hline 3 & Giving medication before or after due time & 57 & 42 & 0 & 1 & 100 \\
\hline 4 & Not diluting a medication that has to be diluted & 67 & 33 & 0 & 0 & 100 \\
\hline 5 & $\begin{array}{l}\text { Not paying attention to proper time of giving a medication (before } \\
\text { and after food) }\end{array}$ & 41 & 51 & 7 & 1 & 100 \\
\hline 6 & $\begin{array}{l}\text { Not taking necessary measures for medications that need special } \\
\text { attention (taking blood pressure and pulse, etc.) }\end{array}$ & 57 & 41 & 2 & 0 & 100 \\
\hline 7 & $\begin{array}{l}\text { Mixing medications(micro set medications) without paying atten- } \\
\text { tion to drug interactions }\end{array}$ & 65 & 32 & 3 & 0 & 100 \\
\hline 8 & Error in injection method in terms of speed of injection. & 36 & 57 & 6 & 1 & 100 \\
\hline 9 & The intravenous injection of an Subcutaneous medication & 62 & 37 & 1 & 0 & 100 \\
\hline 10 & The Subcutaneous injection of an intravenous medication & 55 & 37 & 8 & 0 & 100 \\
\hline 11 & The intramuscular injection of an intravenous medication & 52 & 36 & 12 & 0 & 100 \\
\hline 12 & The intravenous injection of an intramuscular medication & 60 & 39 & 1 & 0 & 100 \\
\hline 13 & Giving patients sublingual or chewable medications orally & 68 & 24 & 8 & 0 & 100 \\
\hline 14 & Giving expired medications to patients & 57 & 32 & 10 & 1 & 100 \\
\hline 15 & Giving postoperative analgesics without physician's prescription & 50 & 34 & 15 & 1 & 100 \\
\hline 16 & Giving the wrong medication to a patient & 55 & 43 & 2 & 0 & 100 \\
\hline 17 & Giving more or less than the prescribed dose & 63 & 28 & 9 & 0 & 100 \\
\hline 18 & $\begin{array}{c}\text { Not paying attention to proper position of patients regarding the } \\
\text { kind of medication }\end{array}$ & 55 & 39 & 6 & 0 & 100 \\
\hline 19 & Not paying attention to effects of drug interactions & 40 & 47 & 13 & 0 & 100 \\
\hline
\end{tabular}

\begin{tabular}{|c|c|c|c|c|c|c|c|}
\hline \multirow{2}{*}{\begin{tabular}{|l} 
No \\
\end{tabular}} & \multirow[t]{2}{*}{ Causes of Refusal to Report Medication Errors } & \multicolumn{5}{|c|}{ Frequency and Percentage of Importance, \% } & \multirow[t]{2}{*}{ Average } \\
\hline & & $\begin{array}{c}\text { Low } \\
\text { important }(1)\end{array}$ & 2 & 3 & 4 & $\begin{array}{c}\text { High } \\
\text { important }(5)\end{array}$ & \\
\hline 1 & Non-significance of occurred error & 23 & 26 & 12 & 10 & 29 & 2.9 \\
\hline 2 & $\begin{array}{l}\text { Nonexistence of recording and reporting medication errors } \\
\text { in the hospital }\end{array}$ & 25 & 12 & 10 & 11 & 42 & 3.3 \\
\hline 3 & $\begin{array}{c}\text { Non significance of reports of medication errors to hospital } \\
\text { authorities }\end{array}$ & 25 & 14 & 14 & 12 & 35 & 3.1 \\
\hline 4 & Fear of being reproached by physician & 22 & 16 & 31 & 21 & 10 & 2.8 \\
\hline 5 & Fear of being reproached by hospital authorities & 26 & 12 & 22 & 24 & 16 & 2.9 \\
\hline 6 & Fear of facing with legal authorities & 33 & 23 & 18 & 14 & 12 & 2.4 \\
\hline 7 & Fear of losing job & 36 & 24 & 11 & 14 & 15 & 2.4 \\
\hline 8 & Fear of being reproached by colleagues & 35 & 26 & 9 & 15 & 15 & 2.5 \\
\hline 9 & $\begin{array}{c}\text { Fear of disclosing the error to the patient and his/her family } \\
\text { and fear of their reaction }\end{array}$ & 28 & 19 & 18 & 18 & 17 & 2.7 \\
\hline 10 & $\begin{array}{c}\text { Fear of consequences and adverse effects of the medication } \\
\text { error }\end{array}$ & 23 & 25 & 16 & 16 & 10 & 2.4 \\
\hline 11 & Disagreement among nurses on the medication error & 22 & 19 & 32 & 24 & 3 & 2.6 \\
\hline 12 & Lack of a clear definition for a medication error & 13 & 24 & 23 & 29 & 11 & 3 \\
\hline 13 & not knowing or diagnosing whether an error has occurred & 19 & 23 & 13 & 29 & 16 & 3 \\
\hline 14 & $\begin{array}{c}\text { Authorities medication errors as individual causes rather } \\
\text { organizational }\end{array}$ & 31 & 21 & 17 & 24 & 7 & 2.5 \\
\hline
\end{tabular}




\section{Discussion}

Because of the effect of medication errors on the increase of mortality rate among patients and increase of hospital expenses, investigation on the errors has gotten a high significance in recent years (9).

In this study, the most important factors in refusal to report medication errors among the nurses under investigation are lack of recording system for medication errors and reporting them to hospital authorities, lack of appropriate feedback, and lack of a clear definition for medication errors, respectively. These findings correspond with findings of other study that indicated administrative factors and the fear of consequences are important barriers of reporting medication errors (10-12).

This issue has also been investigated in other studies. For instance, Mayo and Duncan claimed that $76.9 \%$ of nurses fear from administers and colleagues' reactions (13). Another study claims that given not reporting medication errors, the main concern is disclosing the error to the patient and his/her family and the fear of the legal consequences of the errors are at the second place (14). Considering the findings and the importance of the security of patients, making a positive, effective and stable relationship between nurse managers and nurse staff is going to be necessary. Taking a systemic approach for exploring facilitating factors and tackling barriers and also designing a system for reporting errors especially medication errors are of great significance.

It should be accepted the errors are inevitable; but by reporting and tracking the potentially unwanted errors one can avoid making problems for patients (8). Given the findings, it can be stated that it is necessary for nursing managers in all job categories to provide a secure and appropriate condition for reporting nurses' errors; therefore, nurses can report their errors when they feel secure and when they are sure that the errors will not have adverse consequences for them $(11,15)$. Regarding (formal or informal) reporting of medication errors, investigations conducted in the west show that reporting medication errors has been increasing in recent years (4, 14). This issue needs an urgent attention in Iran, since by tackling the barriers; nurses can be encouraged to report the errors. Revising of working processes such as exact recording and documentation, taking standard procedures, and a proper relationship among the members of medical team will decrease medication errors and rise the reporting of potential errors $(15,16)$. Findings also indicate that high workload, lack of human resources, non-supportiveness of the nature of physical environment, weak relationship between colleagues, and insufficient physical resources are barriers to reporting errors of physicians and nurses, which can be the result of high work pressure and complexity of the process of reporting $(8,17)$. Thus, it is evident that making efforts to tackle the barriers and developing working and intragroup relationships can help reporting probable errors suitably.
However, nurses believe that matters such as knowing that "when an error occurs it has to be reported", applying encouraging method for voluntary and without the name of the nurse, and having a problem solving strategy instead of punishment are positive and effective factors in reporting medication errors $(18,19)$.

Finally, there are different strategies for improving medication error reporting system such as establishing an error reporting system, which does not have punishment programs, improving reporting and communication methods, and providing educational programs pertaining to the importance of medication errors $(20,21)$. Notwithstanding, the role of organizational culture in facilitating the medication error reporting system cannot be denied. Therefore, as Islamic culture is dominant in all Iranian organizations including hospitals, dissemination and improving sincerity and trustworthiness as well as ignoring the errors can be a big help to solve the problems. Future studies should be conducted using interventional designs to identify the major causes of occurring medication errors, other reasons for not reporting them, and strategies to prevent or reduce their occurrence.

This study was carried out on only one hospital and small sample, which can be considered as an important limitation, hampering the results generalizability. Another limitation is the lack of an error reporting system. Therefore, it is suggested that similar studies should be carried out on other public and private hospitals with large samples. Also we believe that this study was limited likelihood of a response bias, because of nurse's reasons for not reporting errors, and it is suggested that to prevent or reduce this situation should be carried out useful strategies.

The results of this study showed that the lack of reporting errors is multifactorial. Thus, to prevent the addition of nurses to other organizational factors such as organizational structure, organizational culture and organizational policies noted therefore, it is recommended that future studies be conducted in the field above.

\section{Acknowledgements}

This study as a part of research project was supported by Shahid Beheshti University of Medical Sciences (Code 1944). The authors kindly acknowledge individuals who participated in this research.

\section{Authors' Contributions}

Davood Mostafaei performed the study and Interviews, extracted concepts from the data and prepared the manuscript. Ahmad Barati Marnani supervised the study and participated in designing and conducting the study, and also manuscript preparation. Haleh Mosavi Esfahani participated in the design of the study, and helped in writing the manuscript. Fatemeh Estebsari contributed in conducting the study. Shiva Shahzaidi participated in manuscript preparation. Ensiyeh Jamshidi helped in writing 
the manuscript. Seyed Samad Aghamiri helped in writing the manuscript. All authors have studied and approved the content of the present manuscript.

\section{Funding/Support}

This study as a part of research project was supported by Shahid Beheshti University of Medical Sciences (Code 1944).

\section{References}

1. Sanghera IS, Franklin BD, Dhillon S. The attitudes and beliefs of healthcare professionals on the causes and reporting of medication errors in a UK Intensive care unit. Anaesthesia. 2007;62(1):53-61.

2. Seki Y, Yamazaki Y. Effects of working conditions on intravenous medication errors in a Japanese hospital. J Nurs Manag. 2006;14(2):128-39.

3. Mrayyan MT, Shishani K, Al-Faouri I. Rate, causes and reporting of medication errors in Jordan: nurses' perspectives. J Nurs Manag. 2007;15(6):659-70.

4. Rogers AE, Dean GE, Hwang WT, Scott LD. Role of registered nurs es in error prevention, discovery and correction. Qual Saf Health Care. 2008;17(2):117-21.

5. Kouhestani H, Baghcheghi N. Refusal in Reporting Medication Errors from the Viewpoints of Nursing Students in Arak University of Medical Sciences. Iranian JfMed Edu. 2009;8(2):285-92.

6. Evans SM, Berry JG, Smith BJ, Esterman A, Selim P, O'Shaughnessy J, et al. Attitudes and barriers to incident reporting: a collaborative hospital study. Qual SafHealth Care. 2006;15(1):39-43.

7. Mohammadnejad E, Ehsani SR, Salari A, Sajjadi A,, HajiesmaeelPour A. Refusal in Reporting Medication Errors from the Perspective of Nurses in Emergency Ward.jgbfnm. 2013;10(1):61-8.

8. Clarke CL. Risk and long-term conditions: the global challenge. $J$ Clin Nurs. 2008;17(5A):1-3.
9. Crossman M. Technical and Environmental Impact on Medication Error in Paramedic Practice: A review of causes, consequences and strategies for prevention. Australasian J Paramedic. 2012;7(3).

10. Keers RN, Williams SD, Cooke J, Ashcroft DM. Causes of medication administration errors in hospitals: a systematic review of quantitative and qualitative evidence. Drug Saf. 2013;36(11):1045-67.

11. Bahadori M, Ravangard R, Aghili A, Sadeghifar J, Gharsi Manshadi M, Smaeilnejad J. The factors affecting the refusal of reporting on medication errors from the nurses' viewpoints: a case study in a hospital in iran. ISRN Nurs. 2013;2013:876563.

12. Tol A, Pourreza A. Assessing the affecting factors on refusal to report medication errors in nursing staffs of Baharlou hospital. Hospital J. 2010;9(2):19-24.

13. Mayo AM, Duncan D. Nurse perceptions of medication errors: what we need to know for patient safety. J Nurs Care Qual. 2004;19(3):209-17.

14. Luk LA, Ng WI, Ko KK, Ung VH. Nursing management of medication errors. Nurs Ethics. 2008;15(1):28-39.

15. Mansouri A, Ahmadvand A, Hadjibabaie M, Kargar M, Javadi M, Gholami K. Types and severity of medication errors in Iran; a review of the current literature. Daru. 2013;21(1):49.

16. Hajibabaee F, Joolaee S, Peyravi H, Haghani H. The relationship of medication errors among nurses with some organizational and demographic characteristics. J Nurs Res. 2011;6(20):83-92.

17. Wilkins K, Shields M. Correlates of medication error in hospitals. Health Rep. 2008;19(2):7-18.

18. Wolf ZR, Hicks R, Serembus JF. Characteristics of medication errors made by students during the administration phase: a descriptive study. J Prof Nurs. 2006;22(1):39-51.

19. Penjoveini S. The study of prevalence and type of medication errors among staff nurses of educational hospitals in Sanandadge. J Nurs Res. 2007;1(1):59-64.

20. Eslamian J, Taheri F, Bahrami M, Mojdeh S. Assessing the nursing error rate and related factors from the view of nursing staff. Iran J Nurs Midwifery Res. 2010;15(Suppl1):272-7.

21. Anderson P, Townsend T. Medication errors: Don't let them happen to you. Americ Nurs Today. 2010;5(3):23-8. 